\title{
Validity assessment of the PROMIS fatigue domain among people living with HIV
}

\author{
L. E. Gibbons ${ }^{1,10^{*}}$, R. Fredericksen' 1 D. S. Batey², L. Dant ${ }^{3}$, T. C. Edwards ${ }^{4}$, K. H. Mayer ${ }^{3,5}$, W. C. Mathews ${ }^{6}$, \\ L. S. Morales ${ }^{7}$, M. J. Mugavero ${ }^{8}$, F. M. Yang ${ }^{9}$, E. Paez ${ }^{6}$, M. M. Kitahata ${ }^{1}$, D. L. Patrick', H. M. Crane ${ }^{1}$, P. K. Crane ${ }^{1}$ \\ and for the Centers for AIDS Research Network of Integrated Clinical Systems (CNICS)
}

\begin{abstract}
Purpose: To evaluate psychometric characteristics and cross-sectional and longitudinal validity of the 7-item PROMIS ${ }^{\circledR}$ Fatigue Short Form and additional fatigue items among people living with HIV (PLWH) in a nationally distributed network of clinics collecting patient reported data at the time of routine clinical care.

Methods: Cross-sectional and longitudinal fatigue data were collected from September 2012 through April 2013 across clinics participating in the Centers for AIDS Research Network of Integrated Clinical Systems (CNICS). We analyzed data regarding psychometric characteristics including simulated computerized adaptive testing and differential item functioning, and regarding associations with clinical characteristics.

Results: We analyzed data from 1597 PLWH. Fatigue was common in this cohort. Scores from the PROMIS ${ }^{\circledR}$ Fatigue Short Form and from the item bank had acceptable psychometric characteristics and strong evidence for validity, but neither performed better than shorter instruments already integrated in CNICS.
\end{abstract}

Conclusions: The PROMIS ${ }^{\circledR}$ Fatigue Item Bank is a valid approach to measuring fatigue in clinical care settings among PLWH, but in our analyses did not perform better than instruments associated with less respondent burden.

Keywords: Fatigue, HIV, PROMIS, Validity, Psychometrics, Measurement, Patient burden

\section{Background}

Fatigue is a common clinical symptom and adversely impacts health-related quality of life. Fatigue is highly prevalent among persons living with HIV (PLWH) $[1,2]$. It is a common side-effect of antiretroviral medications [3], and it is associated with several adverse clinical outcomes, including longer time until depression remission [4], poorer physical functioning $[5,6]$, poorer adherence to antiretroviral medications [7], and virologic failure [8]. Patients rank fatigue as an important domain for providers to know about in order to provide good care [9].

In many cases fatigue is not systematically assessed as part of clinical care. Challenges that impede fatigue assessment for research in PLWH have been outlined

\footnotetext{
*Correspondence: gibbonsl@u.washington.edu

${ }^{10}$ Harborview Medical Center, University of Washington, 325 9th Ave, Box MS 35978, Seattle, WA 98104, USA

Full list of author information is available at the end of the article
}

previously, including lack of consistent measurement, lack of longitudinal measurement, and lack of comprehensive clinical data to examine potential predictors of fatigue [10]. Measuring fatigue for clinical care further compounds these issues as there are substantial time constraints and logistical hurdles that must be addressed to minimize impact of assessment on clinical flow.

One option for assessing fatigue among PLWH is the HIV-Related Fatigue Scale [10-12]. This is a welldesigned measure with 56 items including subscales addressing concepts such as intensity and impact. Unfortunately, it is too long to be useful in most routine clinical care settings. At the opposite end of the spectrum are very brief assessments such as the single item included in the HIV symptoms index [13].

The Patient Reported Outcomes Measurement and Information System (PROMIS ${ }^{\circledR}$, http://www.nihpromis. org) is a National Institutes of Health Roadmap initiative to develop item banks to measure patient-reported 
symptoms. PROMIS investigators developed a fatigue item bank [14]. Items from the bank can be used as either a fixed-length short-form or as a computerized adaptive test (CAT) [15]. The PROMIS ${ }^{\circledR}$ Fatigue Item Bank was developed for people in general rather than specific patient groups such as PLWH, which facilitates comparisons with the general population and across patient groups [16]. Well-developed and calibrated universal fatigue measures could enhance comparability of findings and serve as a common metric of fatigue across conditions [15]. Yet, previous analyses of the PROMIS ${ }^{\circledR}$ fatigue domain were not conducted among PLWH, and were not carried out in the context of routine clinical care. We conducted this study to better understand the properties of the PROMIS ${ }^{\circledR}$ fatigue instrument as part of routine clinical care for PLWH.

\section{Methods}

\section{Study cohort}

This study was conducted in the Centers for AIDS Research Network of Integrated Clinical Systems (CNICS) cohort [17], which integrates comprehensive inpatient and outpatient clinical data on PLWH in the cohort [17]. PLWH complete the CNICS clinical assessment of patient reported measures, symptoms, and outcomes (PROs) every 4-6 months as part of routine clinic visits $[18,19]$. They use touch screen tablets or personal computers using web-based survey software developed specifically for PROs $[18,20]$ to complete the clinical assessment which includes a variety of measures such as the HIV Symptoms Index [13], the Patient Health Questionnaire (PHQ-9) [21, 22] for depression, and the modified Alcohol, Smoking, and Substance Involvement Screening Test [23, 24] for illicit drug use. The assessment was integrated into clinical care for regularly scheduled clinic visits at each site. No exclusions were made on the basis of severe fatigue.

\section{Study participants}

PLWH 18 years old or older who spoke English or Spanish at four clinics (University of Washington Madison HIV Clinic, Seattle; University of Alabama at Birmingham 1917 Clinic, Birmingham; University of California San Diego HIV Clinic, San Diego; and Fenway Health, Boston) were eligible to participate in this study. Data were collected from 1597 PLWH from September 2012 to April 2013.

\section{Qualitative analyses}

We conducted in-depth interviews in English and Spanish with 42 patients endorsing fatigue to elicit concepts regarding the experience of living with fatigue and HIV, as described elsewhere [25]. We excerpted and coded transcribed interview content using codes adapted from PROMIS ${ }^{\circledR}$ Fatigue Item Bank content. We matched coded interview content to bank items. The team assessed unmatched content for possible new item development. We reviewed all proposed items using PROMIS ${ }^{\circledR}$ Qualitative Item Review criteria [26], for readability using the Lexile Analyzer, and for translatability into English or Spanish. We held focus groups with 68 patients and asked them to rank-order the prospective item list in order of importance for their provider to know. We retained the most important items and conducted cognitive interviews with 21 patients to assess item comprehensibility, modifying items as needed [25]. We developed four new items in addition to those already in the PROMIS ${ }^{\circledR}$ Fatigue Item Bank [25].

\section{Item administration}

We administered the 7-item PROMIS ${ }^{\circledR}$ Fatigue Short Form [27], an additional 13 items selected from the PROMIS $^{\circledR}$ Fatigue Item Bank (including four items excluded from the final bank and, thus, without PROMIS ${ }^{\circledR}$ item parameters), and our four new items (see Table 1). We modified response options for five existing PROMIS ${ }^{\circledR}$ items because of qualitative feedback. We used PROMIS ${ }^{\circledR}$ item parameters for all of the other PROMIS ${ }^{\circledR}$ items but calibrated the five items with new response options anew.

\section{Quantitative analyses}

We used Stata [28] for all analyses unless otherwise noted.

\section{Dimensionality}

We used structural equation modeling to determine whether the items were sufficiently unidimensional to use item response theory (IRT) in our sample. All structural equation models were fit in Mplus [29]. We applied the following thresholds for acceptable model fit: confirmatory fit index (CFI) > 0.95, Tucker-Lewis index (TLI) $>0.95$, and root mean squared error of approximation $($ RMSEA) $<0.08$ [30].

\section{PROMIS $^{\circledR}$ item parameters}

We performed additional analyses to determine whether it was appropriate to use PROMIS ${ }^{\circledR}$ item parameters in our population of PLWH. We initially fixed all seven items from the fatigue short form to their PROMIS ${ }^{\circledR}$ values and used modification indices to identify the item for which constraining parameters to PROMIS ${ }^{\circledR}$ values had the greatest impact on model fit. We then removed those constraints and freely estimated parameters for that item and identified the next item that had the greatest impact on model fit. We repeated this procedure until 
Table 1 Fatigue items administered, with a priori subdomains

\begin{tabular}{|c|c|}
\hline Item description & Subdomain \\
\hline \multicolumn{2}{|l|}{ PROMIS 7-item Fatigue Short Form } \\
\hline How often did you feel tired? & Experience \\
\hline How often did you run out of energy? & Experience \\
\hline How often were you too tired to take a bath or shower? & Impact \\
\hline How often did you experience extreme exhaustion? & Experience \\
\hline $\begin{array}{l}\text { How often did your fatigue limit you at work (including } \\
\text { work at home)? }\end{array}$ & Impact \\
\hline How often were you too tired to think clearly? & Impact \\
\hline $\begin{array}{l}\text { How often did you have enough energy to exercise } \\
\text { strenuously? }\end{array}$ & Impact \\
\hline \multicolumn{2}{|l|}{ Other calibrated PROMIS items } \\
\hline How often were you physically drained? & Experience \\
\hline $\begin{array}{l}\text { To what degree did you have to force yourself to get up } \\
\text { and do things because of your fatigue? }\end{array}$ & Impact \\
\hline How run-down did you feel on average? & Experience \\
\hline How fatigued were you on average? & Experience \\
\hline \multicolumn{2}{|c|}{$\begin{array}{l}\text { Items calibrated in PROMIS but administered with different response } \\
\text { options }^{\mathrm{a}}\end{array}$} \\
\hline $\begin{array}{l}\text { How fatigued were you when your fatigue was at its } \\
\text { worst? }\end{array}$ & Experience \\
\hline $\begin{array}{l}\text { To what degree did your fatigue interfere with your } \\
\text { physical functioning? }\end{array}$ & Impact \\
\hline I felt fatigued & Experience \\
\hline I had trouble starting things because I was tired & Impact \\
\hline $\begin{array}{l}\text { How much were you bothered by your fatigue on aver- } \\
\text { age? }\end{array}$ & Experience \\
\hline \multicolumn{2}{|l|}{ Uncalibrated PROMIS items } \\
\hline How often did you wake up feeling exhausted? & Experience \\
\hline $\begin{array}{l}\text { How often did you feel so exhausted that you stayed in } \\
\text { bed all day? }\end{array}$ & Impact \\
\hline $\begin{array}{l}\text { How often were you too exhausted to take your medica- } \\
\text { tion? }\end{array}$ & Impact \\
\hline $\begin{array}{l}\text { How often were you so exhausted that you missed } \\
\text { appointments? }\end{array}$ & Impact \\
\hline \multicolumn{2}{|l|}{ New items from qualitative interviews } \\
\hline $\begin{array}{l}\text { How often were you too exhausted to carry out your } \\
\text { daily responsibilities? }\end{array}$ & Impact \\
\hline How often did your body feel exhausted? & Experience \\
\hline $\begin{array}{l}\text { How often were you too exhausted to chew and swallow } \\
\text { food? }\end{array}$ & Impact \\
\hline How often were you too exhausted to concentrate? & Impact \\
\hline
\end{tabular}

${ }^{a}$ Because different response options were offered in this study, we did not use the PROMIS item parameters for these items

we were left with two anchor items. We extracted factor scores from the PROMIS ${ }^{\circledR}$-fixed model and from a model with the final two anchor items and five freely estimated items and calculated correlations between these scores. We plotted agreement between scores using a variant of a Bland-Altman plot, with the difference between the scores on the $y$-axis and the PROMIS ${ }^{\circledR}$-fixed model scores on the $\mathrm{x}$-axis. We superimposed the standard error of measurement (SEM) curve on this graph and examined whether the differences were smaller than the SEM at each level of fatigue.

\section{Comparison of measurement properties of scores}

We computed an IRT score for all 24 items. We fixed item parameters for the 11 PROMIS $^{\circledR}$ items with PROMIS ${ }^{\circledR}$ response options to their PROMIS ${ }^{\circledR}$ values, so scores are on the PROMIS ${ }^{\circledR}$ metric. We freely estimated parameters for the other 13 items. We compared the SEM for the PROMIS ${ }^{\circledR}-7$ a short form to that from all 24 items.

\section{Simulated CAT}

We used Firestar [31] to simulate CAT from the 24-item bank we administered. We categorized PLWH into groups based on PROMIS ${ }^{\circledR}$ fatigue scores: $<40,40-50,>50-60$, and $>60$. We set the minimum number of items administered by the simulated CAT at 7 and the default stopping rule of SEM $<0.3$ (equivalent: T-metric SEM $<3$ ). We determined the proportion of times each item was administered to people in each fatigue level group. We used seven items as a minimum to determine the extent of overlap between items selected by CAT and items included in the 7-item PROMIS ${ }^{\circledR}$ Fatigue Short Form. As a sensitivity analysis, we performed a second CAT simulation with no minimum number of items and used a 0.3 SEM or seven items maximum stopping rule. We compared patient burden based on the average completion times per item for patients from one site (University of Washington) who completed both instruments, based on the 7-item short form and the number of items in the 2 CAT simulations.

\section{Differential item functioning (DIF)}

We used the Stata command-difwithpar-[32] to evaluate items for DIF with respect to age, sex, race, and nadir CD4 count. We used a $P$ value criterion of 0.05 for uniform and for non-uniform DIF. The-difwithpar-algorithm uses demographic-specific item parameters for items identified with DIF and generates new scores that account for DIF. We evaluated DIF impact by comparing naive scores that ignored DIF to those that accounted for DIF. We use differences of score larger than 0.3 points on the theta metric (larger than 3 points on the T metric) as the primary threshold to indicate salient DIF impact and the median SEM as a more stringent threshold.

\section{Associations with clinical characteristics}

We used Spearman correlations to compare crosssectional associations between clinical characteristics and the HIV Symptoms Index fatigue item [13], the "tired" item from the PHQ-9 [21, 22], the PROMIS ${ }^{\circledR}-7 \mathrm{a}$ score, and the score derived from the entire 24 items we 
administered. The clinical characteristics included: hepatitis $\mathrm{C}$ virus co-infection; nadir and current CD4 count; the number of symptoms endorsed on the HIV Symptoms Index; specific symptoms endorsed on the HIV Symptoms Index; quality of life estimated using EQ-5D responses [33-36]; and the total PHQ-9 score. Among PLWH taking antiretroviral medications for HIV, we also determined associations between fatigue scores and medication adherence based on the last time the person stated they had missed medications, their self-reported ability to take medications, and the proportion of medications they were estimated to have taken [37-42].

\section{Test-retest reliability}

We had 51 people return to clinic on a second occasion from 6-14 days following their initial assessment to repeat the assessment. Since this involved an extra visit outside the context of clinical care, we provided an incentive of $\$ 15$ for this activity. We used intraclass correlation coefficients (ICCs) to measure test-retest reliability.

\section{Longitudinal evaluation}

A subset of 249 PLWH had repeat assessment on a second routine clinical care visit from 79-203 days following their initial assessment [median 119 days, interquartile range (IQR) 105-134 days]. Given the episodic nature of HIV symptoms [43], we were interested first in describing changes in fatigue. We also sought to compare changes in fatigue measures in two situations where change might be expected: concurrent with a change in depression symptoms or a change in methamphetamine use on the clinical assessment.

\section{Results}

\section{Demographic and clinical characteristics from the cross-sectional quantitative data}

English questionnaires were completed by 1597 PLWH (Table 2); we included Spanish speakers in our qualitative analyses, but there were too few respondents in Spanish $(\mathrm{n}=94)$ for meaningful quantitative analyses. Mean age (SD) was 45.7 (10.4), with a range from 20 to 83 (IQR 39, 53).

Fatigue was common in this cohort. Using the HIV Symptoms Index single item, 65\% stated they had fatigue (Table 2). Scores from the PROMIS ${ }^{\circledR}$ items mapped closely to these scores from the HIV Symptoms Index. As shown in Fig. 1, median fatigue scores on the PROMIS ${ }^{\circledR}$ metric ranged from just below 40 for those who stated they did not have fatigue to just over 65 for those who stated they had fatigue and that it bothered them a lot.

\section{Dimensionality}

A single factor confirmatory factor analysis model did not fit well by RMSEA criteria (CFI 0.98, TLI 0.98, RMSEA
0.103). We assigned items a priori to one of two subdomains, the experience of fatigue vs. the impact of fatigue, based on PROMIS ${ }^{\circledR}$ 's domain framework (see Table 1), but this model did not fit well and had loadings that did not support the theoretical structure, such as negative loadings on a subdomain. A negative loading means that as levels of the item were of increasing severity, the level of fatigue impact was expected to be lower down, which is difficult to explain.

We then considered modification indices from a single factor model that suggested candidate pairs of items with residual correlations that would have the greatest impact on model fit. We included 6 such pairs, which resulted in a model with CFI 0.99, TLI 0.99, and RMSEA 0.08. We extracted factor scores for the single factor score and the bifactor score with the six residual correlations. These scores were highly correlated at 0.9999 . We compared standardized factor loadings between these models, and the largest difference was 0.020 , well lower than the 0.10 threshold that would indicate a salient difference in loadings between the single factor and bifactor models [44]. These findings led us to conclude that the items were sufficiently unidimensional to proceed with IRT analyses.

\section{PROMIS item parameters}

The loadings and thresholds for the two anchor items and the five freely estimated items are shown in Additional file 1 . The correlation between the score using those parameters and the score based entirely on PROMIS parameters was $>0.99$. All of the score differences were within the SEM curve thresholds (Additional file 2). These results supported use of PROMIS item parameters for PLWH.

\section{Measurement properties}

We show a plot of the SEM for the 24 items administered and for the 7-item PROMIS ${ }^{\circledR}$ Fatigue Short Form subset in Fig. 2. The median SEM was 0.29 (range 0.240.57; IQR 0.26-0.34) for the 7-item PROMIS ${ }^{\circledR}$ Fatigue Short Form and 0.15 (range 0.11-0.52; IQR 0.14-0.20) using all 24 items. On the T-score metric, the 7-item PROMIS ${ }^{\circledR}$ Fatigue Short Form has an SEM $<3$ over the 45-73 range, while using all 24 items gives an SEM under 3 for all scores 35 and above. We also show a histogram of observed fatigue scores from the 7-item PROMIS ${ }^{\circledR}$ Fatigue Short Form on the same plot. There are very few people with extremely high levels of fatigue (over 73) for whom the 24 items would provide a markedly improved level of precision; most of the people for whom differences in precision between the 7-item short form and the 24-item bank are characterized by low levels of fatigue, with scores $35-45$ on the PROMIS ${ }^{\circledR}$ metric. While scores in this range are common, it may not be clinically 
Table 2 Participant characteristics $(n=1597)$

\begin{tabular}{|c|c|c|c|c|c|c|}
\hline \multirow[t]{2}{*}{ Characteristic } & \multirow[t]{2}{*}{$\mathbf{N}$} & \multirow[t]{2}{*}{ Percent } & \multicolumn{2}{|c|}{ Recent CD4 $<500$} & \multicolumn{2}{|c|}{ Recent CD4 500+ } \\
\hline & & & $\mathbf{N}$ & Percent & $\mathbf{N}$ & Percent \\
\hline Male & 1315 & 82.3 & 591 & 84.8 & 724 & 80.4 \\
\hline \multicolumn{7}{|l|}{ Race } \\
\hline White & 861 & 53.9 & 346 & 49.6 & 515 & 57.2 \\
\hline Black & 461 & 28.9 & 197 & 28.3 & 264 & 29.3 \\
\hline Hispanic & 195 & 12.2 & 105 & 15.1 & 90 & 10.0 \\
\hline Other & 80 & 5.0 & 49 & 7.0 & 31 & 3.4 \\
\hline \multicolumn{7}{|c|}{ HIV Symptom Index fatigue item } \\
\hline No fatigue & 558 & 34.9 & 233 & 33.4 & 325 & 36.1 \\
\hline Doesn't bother me & 151 & 9.5 & 66 & 9.5 & 85 & 9.4 \\
\hline Bothers me a little & 383 & 24.0 & 161 & 23.1 & 222 & 24.7 \\
\hline Bothers me & 260 & 16.3 & 117 & 16.8 & 143 & 15.9 \\
\hline Bothers me a lot & 221 & 13.8 & 110 & 15.8 & 111 & 12.3 \\
\hline Did not answer & 24 & 1.5 & 10 & 1.4 & 14 & 1.6 \\
\hline \multicolumn{7}{|l|}{ CD4 Nadir } \\
\hline$<200$ & 732 & 45.8 & 487 & 69.9 & 245 & 27.2 \\
\hline 200 to $<350$ & 392 & 24.6 & 152 & 21.8 & 240 & 26.7 \\
\hline 350 or higher & 468 & 29.3 & 55 & 7.9 & 413 & 45.9 \\
\hline Missing & 5 & 0.3 & 3 & 0.4 & 2 & 0.2 \\
\hline Taking anti-HIV meds & 1412 & 88.4 & 604 & 86.7 & 808 & 89.8 \\
\hline
\end{tabular}

important to measure fatigue levels precisely in these individuals 1.5-2.5 SD below national norms for fatigue.

\section{CAT results}

Our first CAT simulation used a minimum of seven items. With this criterion, only people with PROMIS fatigue scores $<40$ required more than seven items to achieve a SEM $<3$ on the T-metric (Table 3). There were two items that were administered in all simulated CATs: "How run-down did you feel on average" and "How fatigued were you on average." The item "How often were you physically drained" was almost always administered. None of the items from the 7-item PROMIS ${ }^{\circledR}$ Fatigue Short Form was routinely selected for CAT administration across all fatigue levels, though "How often did you feel tired" and "How often did you run out of energy" were always administered to individuals with fatigue scores $<40$.

As outlined in our previous publication, we developed four new fatigue items based on our qualitative work [25]. In simulated CAT, one of these, "How often did your body feel exhausted?" was selected $46 \%$ of the time; it was always selected for people with fatigue scores $\leq 40,61 \%$ of the time for fatigue scores $>40-50$ and $46 \%$ of the time for those with the highest levels of fatigue. In contrast, the other new items we developed were either never or rarely selected for people with fatigue levels $>40$; these items were "How often were you too exhausted to carry out your daily responsibilities?", "How often were you too exhausted to chew and swallow food?" and "How often were you too exhausted to concentrate?"

In our secondary analyses, we completed another CAT simulation with no minimum number of items and a stopping rule of either a standard error of measurement $<3$ points on the $\mathrm{T}$ metric or up to 7 items maximum; the median (IQR) number of items administered was $3(3-4)$.

Based on the mean time per item for the PROMIS fatigue items (mean $6.73 \mathrm{~s}$, SD 2.74 per item), a person completing the 7-item PROMIS short form or 7-item CAT would be expected to take an average of $47.1 \mathrm{~s}$. Based on the second simulated CAT where people completed a mean of 3 items, the average completion time for the PROMIS fatigue CAT would be $20.2 \mathrm{~s}$. This is in comparison to an estimated time to complete the HIV Symptom Index fatigue screening item of $6 \mathrm{~s}$ (mean $6.0 \mathrm{~s}$, SD 10.1).

\section{DIF results}

A few items had DIF with respect to age, sex, race, and/ or nadir CD4 count with the very sensitive DIF thresholds we used (results not shown). There was negligible DIF impact, and for none of these covariates was there any individual PLWH where accounting for DIF 


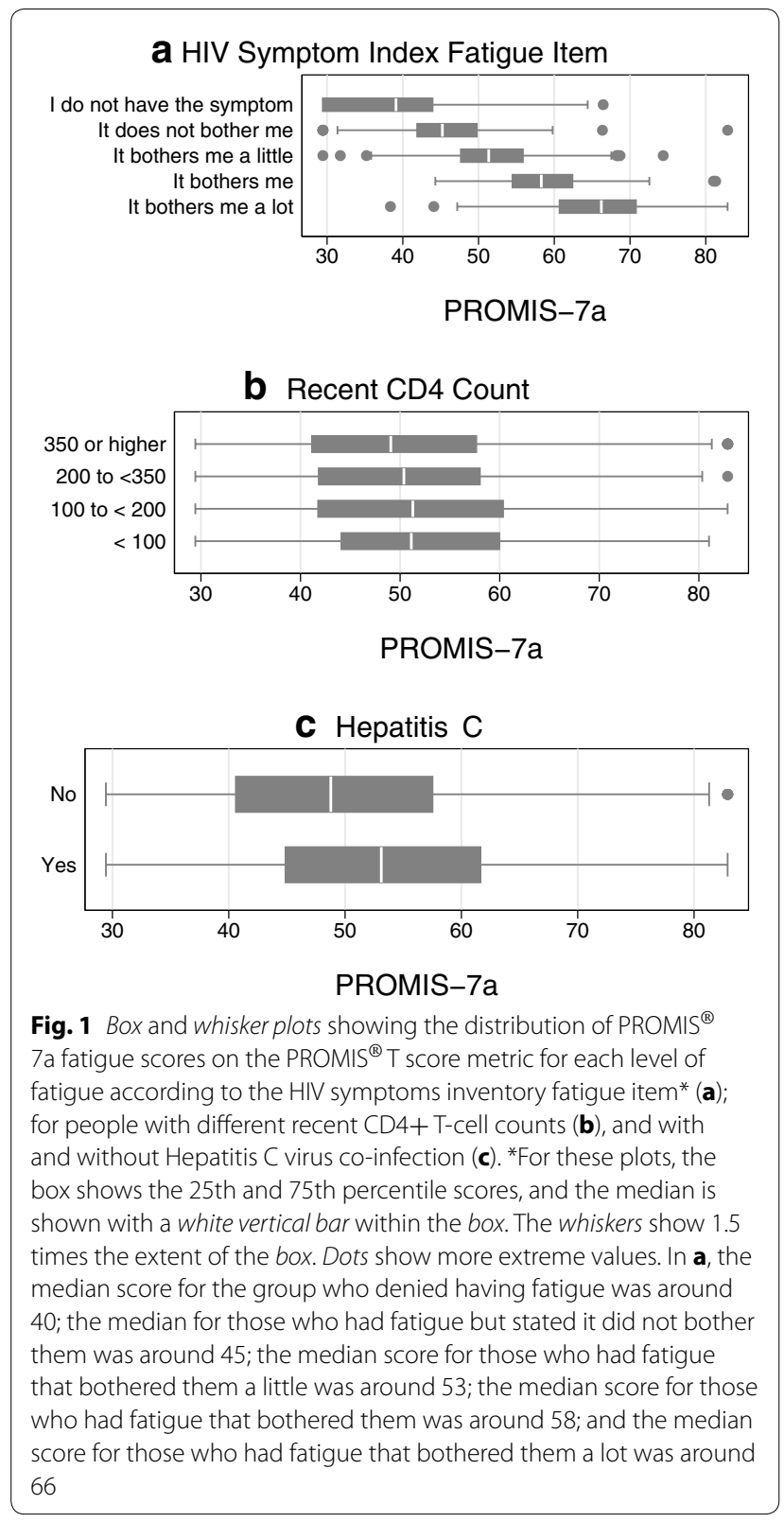

led to a change in score as much as three points on the PROMIS T-score metric. Indeed, when we considered a more stringent 1.7 points PROMIS T-score metric (the median SEM for this sample), only $1-7$ people (all $<1 \%$ ) had DIF impact of this magnitude with respect to each of these covariates. We concluded that there was negligible DIF in these items with respect to these covariates.

\section{Associations with clinical characteristics}

The HIV Symptom Index single item fatigue score was closely associated with the 7 -item PROMIS ${ }^{\circledR}$ Fatigue Short Form $(\rho=0.82)$ and with the score from all of 24

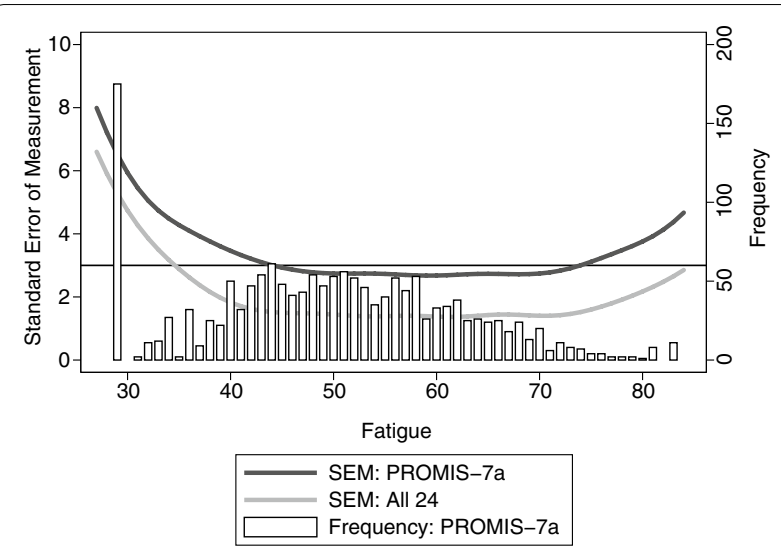

Fig. 2 Histogram of observed fatigue levels (open bars) superimposed on standard error of measurement curves for the 24 fatigue items administered to participants (lower light gray curve) and for the 7-item PROMIS ${ }^{\circledR}$ Fatigue Short Form (upper darker gray curve). A horizontal line is at a SEM of 3, which is the common default stopping rule for computerized adaptive testing

items $(\rho=0.85)$ (Table 4). Similarly, the PHQ-9 fatigue item was closely associated with the HIV Symptoms Index fatigue item $(\rho=0.77)$, with the 7 -item PROMIS ${ }^{\circledR}$ Fatigue Short Form $(\rho=0.75)$, and with the 24-item score $(\rho=0.77)$. Correlations with clinical characteristics were generally as strong for the HIV Symptom Index fatigue item as they were for either the 7-item PROMIS ${ }^{\circledR}$ Fatigue Short Form or the full 24-item score.

\section{Test-retest reliability}

Fifty-one people completed the 7-item PROMIS ${ }^{\circledR}$ Fatigue Short Form again 6-14 days later (median 8, IQR 7-11 days). The ICC was $0.74(0.55,0.83)$. The mean change was -0.17 points, though 4 people had a decrease of at least on point and 2 had an increase of at least one point, either due to true changes in fatigue [43] or measurement error. Among the 31 people who said their level of fatigue was "about the same" as previously, the ICC was similar at $0.66(0.44,0.81)$.

\section{Longitudinal analyses}

On average there was little change in level of fatigue over approximately 4 months-the mean change was -0.16 . However, this obscures individual variation, in that $9 \%$ reported an increase in fatigue of at least one point, and $16 \%$ reported a decrease of at least one point. Changes in the PHQ-9 depression score were more highly correlated with changes in the HIV Symptom Index fatigue item (Spearman $\rho=0.47$ ) than were changes in the 7-item PROMIS $^{\circledR}$ Fatigue Short Form score $(\rho=0.39)$. Only 13 people changed from using methamphetamines to not, or vice versa, so comparisons of the fatigue measures were not feasible. 
Table 3 Frequency of item administration in simulated computerized adaptive testing, by level of fatigue

\begin{tabular}{|c|c|c|c|c|c|}
\hline \multirow[t]{2}{*}{ Item } & \multirow[t]{2}{*}{ Overall $(n=1597)$} & \multicolumn{4}{|l|}{ Fatigue score } \\
\hline & & $\leq 40(n=246)$ & $>40-50(n=561)$ & $>50-60(n=556)$ & $>60(n=234)$ \\
\hline How often did you feel tired? & 22.0 & 100.0 & 18.4 & 0.0 & 1.3 \\
\hline How often did you run out of energy? & 57.5 & 100.0 & 54.2 & 45.9 & 48.7 \\
\hline $\begin{array}{l}\text { How often were you too tired to take a bath or } \\
\text { shower? }\end{array}$ & 6.6 & 43.1 & 0.0 & 0.0 & 0.0 \\
\hline How often did you experience extreme exhaustion? & 7.3 & 45.9 & 0.0 & 0.0 & 1.7 \\
\hline $\begin{array}{l}\text { How often did your fatigue limit you at work (includ- } \\
\text { ing work at home)? }\end{array}$ & 8.9 & 57.7 & 0.0 & 0.0 & 0.0 \\
\hline How often were you too tired to think clearly? & 7.8 & 45.9 & 0.0 & 0.0 & 4.7 \\
\hline $\begin{array}{l}\text { How often did you have enough energy to exercise } \\
\text { strenuously? }\end{array}$ & 8.7 & 56.5 & 0.0 & 0.0 & 0.0 \\
\hline How often were you physically drained? & 99.7 & 100.0 & 100.0 & 100.0 & 97.9 \\
\hline $\begin{array}{l}\text { To what degree did you have to force yourself to get } \\
\text { up and do things? }\end{array}$ & 79.8 & 46.7 & 69.5 & 100.0 & 91.5 \\
\hline How run-down did you feel on average? & 100.0 & 100.0 & 100.0 & 100.0 & 100.0 \\
\hline How fatigued were you on average? & 100.0 & 100.0 & 100.0 & 100.0 & 100.0 \\
\hline $\begin{array}{l}\text { How fatigued were you when your fatigue was at its } \\
\text { worst? }\end{array}$ & 11.6 & 75.2 & 0.0 & 0.0 & 0.0 \\
\hline $\begin{array}{l}\text { To what degree did your fatigue interfere with your } \\
\text { physical functioning? }\end{array}$ & 70.6 & 49.6 & 38.3 & 100.0 & 100.0 \\
\hline I felt fatigued & 47.3 & 64.2 & 82.7 & 22.1 & 4.3 \\
\hline I had trouble starting things because I was tired & 20.5 & 48.0 & 0.0 & 35.1 & 6.0 \\
\hline $\begin{array}{l}\text { How much were you bothered by your fatigue on } \\
\text { average? }\end{array}$ & 85.2 & 85.4 & 75.6 & 89.9 & 97.0 \\
\hline How often did you wake up feeling exhausted? & 7.6 & 49.2 & 0.0 & 0.0 & 0.0 \\
\hline $\begin{array}{l}\text { How often did you feel so exhausted that you stayed } \\
\text { in bed all day? }\end{array}$ & 6.9 & 44.7 & 0.0 & 0.0 & 0.0 \\
\hline $\begin{array}{l}\text { How often were you too exhausted to take your } \\
\text { medication? }\end{array}$ & 6.2 & 40.2 & 0.0 & 0.0 & 0.0 \\
\hline $\begin{array}{l}\text { How often were you so exhausted that you missed } \\
\text { appointments? }\end{array}$ & 6.6 & 42.7 & 0.0 & 0.0 & 0.0 \\
\hline $\begin{array}{l}\text { How often were you too exhausted to carry out your } \\
\text { daily responsibilities? }\end{array}$ & 7.0 & 43.9 & 0.0 & 0.0 & 1.7 \\
\hline How often did your body feel exhausted? & 46.1 & 100.0 & 61.3 & 7.0 & 46.2 \\
\hline $\begin{array}{l}\text { How often were you too exhausted to chew and } \\
\text { swallow food? }\end{array}$ & 6.1 & 39.8 & 0.0 & 0.0 & 0.0 \\
\hline How often were you too exhausted to concentrate? & 7.0 & 45.5 & 0.0 & 0.0 & 0.0 \\
\hline Mean number of items & 8.3 & 15.2 & 7.0 & 7.0 & 7.0 \\
\hline Mean standard error of measurement (T-metric) & 2.2 & 3.2 & 2.1 & 2.0 & 2.1 \\
\hline
\end{tabular}

\section{Discussion}

In a thorough evaluation of the psychometric properties of the 7-item PROMIS ${ }^{\circledR}$ Fatigue Short Form and additional items selected from the PROMIS ${ }^{\circledR}$ Fatigue Item Bank or items specifically developed for this project, we found that these fatigue items had excellent content validity among PLWH. While the 24 fatigue items did not form a scale that was strictly unidimensional, it was sufficiently unidimensional to use item response theory. Furthermore, our analyses suggested that PROMIS ${ }^{\circledR}$ item parameters were appropriate to use among PLWH. We used very sensitive DIF detection thresholds and identified items with DIF, but did not find salient impact for DIF with respect to age, sex, race, or nadir CD4 count. Scores from the 7 -item PROMIS ${ }^{\circledR}$ Fatigue Short Form or from all 24 items from the fatigue item bank had excellent validity in a variety of analyses, but were no better than the HIV Symptom Index single fatigue item measure or the fatigue item from the PHQ-9. The HIV Symptom Index single fatigue item has limited ability to detect change over time, because it has only a few response options. Nevertheless, in the longitudinal sample, we did 
Table 4 Spearman correlation coefficients between fatigue measures and clinical characteristics

\begin{tabular}{|c|c|c|c|c|c|c|c|c|}
\hline & \multicolumn{2}{|c|}{ HIVSI fatigue } & \multicolumn{2}{|c|}{ PHQ-9"tired" } & \multicolumn{2}{|l|}{ PROMIS-7a } & \multicolumn{2}{|l|}{ All 24 items } \\
\hline & Coefficient & $P$ value & Coefficient & $P$ value & Coefficient & P value & Coefficient & $P$ value \\
\hline \multicolumn{9}{|l|}{ Factors related to fatigue itself } \\
\hline HIV Symptom Index 1-item Fatigue response & - & - & 0.77 & $<0.0001$ & 0.82 & $<0.0001$ & 0.85 & $<0.0001$ \\
\hline PHQ-9 item "feeling tired or having little energy" & - & - & - & - & 0.75 & $<0.0001$ & 0.77 & $<0.0001$ \\
\hline Hepatitis-C & 0.09 & 0.0004 & 0.09 & 0.0002 & 0.11 & $<0.0001$ & 0.12 & $<0.0001$ \\
\hline \multicolumn{9}{|l|}{ HIV disease severity } \\
\hline Current CD4 count & -0.04 & 0.1402 & -0.04 & 0.1562 & -0.05 & 0.0699 & -0.04 & 0.2392 \\
\hline CD4 nadir & 0.01 & 0.8828 & 0.05 & 0.0534 & 0.00 & 0.8584 & 0.01 & 0.3481 \\
\hline \multicolumn{9}{|l|}{ Symptom burden } \\
\hline Number of symptoms in inventory & 0.69 & $<0.0001$ & 0.59 & $<0.0001$ & 0.64 & $<0.0001$ & 0.66 & $<0.0001$ \\
\hline \multicolumn{9}{|l|}{ Specific symptoms } \\
\hline Fever, chills or sweats & 0.42 & $<0.0001$ & 0.37 & $<0.0001$ & 0.64 & $<0.0001$ & 0.44 & $<0.0001$ \\
\hline Dizzy or lightheaded & 0.51 & $<0.0001$ & 0.46 & $<0.0001$ & 0.42 & $<0.0001$ & 0.54 & $<0.0001$ \\
\hline Pain/numbness/tingling in hands/feet & 0.44 & $<0.0001$ & 0.35 & $<0.0001$ & 0.52 & $<0.0001$ & 0.49 & $<0.0001$ \\
\hline Having difficulty remembering & 0.57 & $<0.0001$ & 0.48 & $<0.0001$ & 0.46 & $<0.0001$ & 0.62 & $<0.0001$ \\
\hline Nausea or vomiting & 0.45 & $<0.0001$ & 0.37 & $<0.0001$ & 0.63 & $<0.0001$ & 0.47 & $<0.0001$ \\
\hline Diarrhea or loose bowel movements & 0.41 & $<0.0001$ & 0.34 & $<0.0001$ & 0.47 & $<0.0001$ & 0.42 & $<0.0001$ \\
\hline Feeling sad or depressed & 0.65 & $<0.0001$ & 0.55 & $<0.0001$ & 0.40 & $<0.0001$ & 0.66 & $<0.0001$ \\
\hline Feeling nervous or anxious & 0.57 & $<0.0001$ & 0.50 & $<0.0001$ & 0.63 & $<0.0001$ & 0.61 & $<0.0001$ \\
\hline Difficulty falling or staying asleep & 0.57 & $<0.0001$ & 0.50 & $<0.0001$ & 0.58 & $<0.0001$ & 0.59 & $<0.0001$ \\
\hline Coughing or trouble catching breath & 0.39 & $<0.0001$ & 0.36 & $<0.0001$ & 0.56 & $<0.0001$ & 0.44 & $<0.0001$ \\
\hline Headaches & 0.42 & $<0.0001$ & 0.35 & $<0.0001$ & 0.43 & $<0.0001$ & 0.46 & $<0.0001$ \\
\hline Appetite loss/change taste of food & 0.45 & $<0.0001$ & 0.43 & $<0.0001$ & 0.44 & $<0.0001$ & 0.52 & $<0.0001$ \\
\hline Bloating pains or gas in stomach & 0.44 & $<0.0001$ & 0.37 & $<0.0001$ & 0.51 & $<0.0001$ & 0.47 & $<0.0001$ \\
\hline Muscle aches or joint pain & 0.47 & $<0.0001$ & 0.42 & $<0.0001$ & 0.47 & $<0.0001$ & 0.52 & $<0.0001$ \\
\hline Sex-loss of interest/satisfaction & 0.47 & $<0.0001$ & 0.41 & $<0.0001$ & 0.50 & $<0.0001$ & 0.50 & $<0.0001$ \\
\hline Body changes-fat deposits/wt gain & 0.46 & $<0.0001$ & 0.38 & $<0.0001$ & 0.50 & $<0.0001$ & 0.49 & $<0.0001$ \\
\hline Weight loss or wasting & 0.32 & $<0.0001$ & 0.30 & $<0.0001$ & 0.49 & $<0.0001$ & 0.39 & $<0.0001$ \\
\hline EQ-5D & 0.59 & $<0.0001$ & 0.56 & $<0.0001$ & 0.38 & $<0.0001$ & 0.64 & $<0.0001$ \\
\hline PHQ-9 & 0.75 & $<0.0001$ & 0.84 & $<0.0001$ & 0.63 & $<0.0001$ & 0.77 & $<0.0001$ \\
\hline Self-rated health (EuroQOL) & -0.53 & $<0.0001$ & 0.50 & $<0.0001$ & 0.74 & $<0.0001$ & -0.57 & $<0.0001$ \\
\hline \multicolumn{9}{|l|}{ Among ART users } \\
\hline Last time missed meds & -0.15 & $<0.0001$ & -0.16 & $<0.0001$ & -0.18 & $<0.0001$ & -0.19 & $<0.0001$ \\
\hline Ability to take meds & -0.19 & $<0.0001$ & -0.24 & $<0.0001$ & -0.23 & $<0.0001$ & -0.23 & $<0.0001$ \\
\hline Take how much of meds & -0.14 & 0.0001 & -0.16 & $<0.0001$ & -0.17 & $<0.0001$ & -0.17 & $<0.0001$ \\
\hline
\end{tabular}

not find evidence that the PROMIS scores were more responsive to change than was the HIV Symptom Index fatigue item or the PHQ-9 fatigue item.

Fatigue is clearly a relevant consideration for this clinical population. Sizable numbers of PLWH had substantial levels of fatigue. One advantage of the PROMIS ${ }^{\circledR}$ fatigue metric is that we can relate fatigue levels to national averages. As shown in Fig. 1 and in Table 3, substantial numbers of PLWH endorse high levels of fatigue. Those who stated that they have fatigue that bothers them a lot on the HIV Symptom Index have median (IQR) PROMIS ${ }^{\circledR}$ fatigue scores of 66 (IQR 61-71), which is about 1.5 SD (1-2 SD) above the national average.
Our CAT simulations showed a small amount of overlap with the 7-item PROMIS ${ }^{\circledR}$ Fatigue Short Form. We set up the first simulation such that each individual received at least seven items to facilitate comparisons with the short form. Only people with very low fatigue levels received more than 7 items from the simulated CAT; everyone else received exactly 7 items. While the 7-item short form may not include the most informative items from the PROMIS ${ }^{\circledR}$ Fatigue Item Bank, it nevertheless had good measurement precision across a broad range of fatigue levels (see Fig. 2). Furthermore, the 7-item PROMIS ${ }^{\circledR}$ Fatigue Short Form performed well in all of our validity analyses; indeed, scores from 
the 7-item PROMIS ${ }^{\circledR}$ Fatigue Short Form performed just as well as scores from the entire 24 items we considered. At the same time, briefer instruments, including the fatigue item from the PHQ-9 and the single HIV Symptom Index fatigue item, also did well in all of our validity analyses. We did not find a compelling case to choose the PROMIS $^{\circledR}$ fatigue scores over much shorter instruments. A CAT with different specifications could have arrived at a PROMIS fatigue score in fewer items, but it would be unlikely to have better performance in our validity analyses than the entire scale considered here. Furthermore, the HIV Symptom Index fatigue item required much less time on average for patients to complete than the 7-item PROMIS short form, CAT, or even the shorter CAT with an average of 3 items. While this may be of limited importance in research settings, minimizing patient burden in clinical care settings is important to avoid impacting clinical flow.

Our findings should be considered in the context of strengths and limitations. Our study was performed in CNICS, which is a nationally distributed cohort of PLWH who are in clinical care. Our data were collected from convenience samples of PLWH seen in particular calendar months, and were not purposefully sampled from people particularly likely to have changing fatigue levels. Generalizability is limited as our study was conducted only among PLWH. We did administer the PROMIS fatigue items to Spanish speakers, but had too few of them during the data collection window to facilitate analyses of DIF. We found no evidence of DIF with respect to four covariates, but were not able to evaluate DIF with respect to Spanish vs. English. The CNICS assessment of patient-reported measures now includes Amharic, but unfortunately, an Amharic version of the PROMIS Fatigue Item Bank has not been developed, nor were we able to assess the performance of these items in any other language.

Our ability to evaluate change in fatigue over time was limited, because we had few options for external comparison. One validation option was changes in depression levels as measured by the PHQ-9, where we found that the HIV Symptom Index fatigue item was more closely correlated to changes in depression levels than were PROMIS scores. In theory, IRT scores are more accurate measures of change over time than ordinal scales, because they have linear measurement properties [45], which means that one point of change in a score corresponds to the same amount of change in fatigue regardless of the initial level of fatigue. Indeed, PROMIS ${ }^{\circledR}$ scores may have shown better responsiveness to change than the HIV symptoms index fatigue item scores had we designed our study specifically to collect data on people expected to change [46]. In that setting, a brief CAT may prove to have better responsiveness to change than the single HIV symptoms index fatigue item and may fit in a reasonable time footprint, making this a feasible choice in routine clinical care settings. Firmer conclusions regarding responsiveness of PROMIS ${ }^{\circledR}$ scores among PLWH will require additional data.

This study has several strengths that are also worth noting. It includes a particularly relevant population (PLWH) given the high rates of fatigue experienced by a substantial proportion of this group. We studied the performance of these items in a geographically and racially/ ethnically diverse population. We performed a variety of psychometric analyses using state-of-the-art approaches.

Fatigue in PLWH often does not remit [10], suggesting the need for additional research to better understand factors leading to fatigue in PLWH and interventions to successfully address it. Research on fatigue among PLWH will require a sustainable systematic approach to measuring fatigue in clinical care.

\section{Conclusions}

The PROMIS ${ }^{\circledR}$ Fatigue Short Form and other fatigue items performed well among PLWH, though we did not find evidence that they performed better than shorter legacy scales in the specific context of routine clinical care. Unless comparison to national norms is needed, the HIV Symptom Index fatigue item may be preferred in HIV clinical care settings due to reduced patient burden.

\section{Additional files}

Additional file 1. Loadings and thresholds for Mplus based on the PROMIS item bank parameters, and using 2 anchor items and 5 freely estimated items.

Additional file 2. Difference in scores between the PROMIS-7a scored using PROMIS item parameters and a score where 2 items are fixed to the PROMIS item parameters and the other 5 are freely estimated. The horizontal line at zero represents no difference, and the upper and lower curves represent the standard error of measurement. All differences are within the standard error of measurement curves.

\section{Abbreviations}

CAT: computerized adaptive test; CFI: confirmatory fit index; CNICS: Centers for AIDS Research Network of Integrated Clinical Systems; DIF: differential item functioning; ICC: intraclass correlation coefficient; IQR: interquartile Range; IRT: item response theory; $\mathrm{PHQ}-9$ : patient health questionnaire for depression; PLWH: persons living with HIV; PROMIS: Patient Reported Outcomes Measurement and Information System; PROs: patient reported measures, symptoms, and outcomes; RMSEA: root mean squared error of approximation; SEM: standard error of measurement; TLI:Tucker-Lewis Index.

\section{Authors' contributions}

LG conducted the statistical analyses and drafted sections of the manuscript. RF conducted all the qualitative analyses and made substantial contributions to the conception and design of the study and acquisition of data. DB, $L D, K M, W M, M M, E P$, and MK were involved in acquisition of data and critical revision of the manuscript for important intellectual content. TE, LM, and FY, were involved in critical revision of the manuscript for important intellectual 
content. DP was involved in the conception and design of the study and critical revision of the manuscript for important intellectual content. HC made substantial contributions to the conception and design of the study and acquisition of data and provided important intellectual content. PC made substantial contributions to the conception and design of the study and drafted sections of the manuscript. All authors read and approved the final manuscript.

\begin{abstract}
Author details
${ }^{1}$ Department of Medicine, Harborview Medical Center, University of Washington, 325 9th Ave, Box 359931, Seattle, WA, USA. ${ }^{2}$ Department of Social Work, University of Alabama at Birmingham, 900 13th Street S, Suite 203, Birmingham, AL 35294, USA. ${ }^{3}$ Fenway Institute, 1340 Boylston Street, Boston, MA 02215, USA. ${ }^{4}$ Department of Health Services, University of Washington, 1208 NE 43rd Street, Seattle, WA 98195, USA. ${ }^{5}$ Department of Medicine, Harvard Medical School, Boston, MA, USA. ${ }^{6}$ Department of Medicine, University of California at San Diego, 4168 Front Street, San Diego, CA 92103, USA. ${ }^{7}$ Center for Health Equity, Diversity and Inclusion, School of Medicine, University of Washington, 1959 Pacific Street, Seattle, WA 98195, USA. ${ }^{8}$ Department of Medicine, University of Alabama at Birmingham, 908 20th Street S, Suite 250, Birmingham, AL 35205, USA. ${ }^{9}$ Department of Biostatistics and Epidemiology, Augusta University, 1120 15th Street, AE-1035, Augusta, GA 30912, USA. ${ }^{10}$ Harborview Medical Center, University of Washington, 325 9th Ave, Box MS 35978, Seattle, WA 98104, USA.
\end{abstract}

\section{Acknowledgements}

We would like to thank the patients and clinics across CNICS. This research was funded by a cooperative agreement from the National Institute of Allergy and Infectious Diseases (NIAID) and National Institute of Arthritis and Musculoskeletal and Skin Diseases (NIAMS) (Grant \#U01 AR057954). Support was also provided by the National Institute of Allergy and Infectious Diseases (NIAID) University of Washington Center for AIDS Research (Grant \#P30 Al027757) and CNICS (R24 Al067039).

\section{Competing interests}

The authors declare that they have no competing interests.

\section{Availability of data and materials}

Deidentified data that support the findings of this study are available from CNICS (https://www.uab.edu/cnics/) with a concept proposal approved by the CNICS research coordination committee. The data are not publicly available due to them containing information that could compromise research participant privacy/consent.

\section{Ethical approval and consent to participate}

All procedures performed in studies involving human participants were in accordance with the ethical standards of the institutional and/or national research committee and with the 1964 Helsinki declaration and its later amendments or comparable ethical standards. Informed consent was obtained from all individual participants included in the study. Study procedures were approved by Institutional Review Boards at each site (CNICS Data Repository \#27647).

\section{Funding}

This research was funded by a cooperative agreement from the National Institute of Allergy and Infectious Diseases (NIAID) and National Institute of Arthritis and Musculoskeletal and Skin Diseases (NIAMS) (Grant \#U01 AR057954). Support was also provided by the National Institute of Allergy and Infectious Diseases (NIAID) University of Washington Center for AIDS Research (Grant \#P30 Al027757) and CNICS (R24 Al067039).

Received: 30 January 2017 Accepted: 19 March 2017

Published online: 11 April 2017

\section{References}

1. Jong E, Oudhoff LA, Epskamp C, et al. Predictors and treatment strategies of HIV-related fatigue in the combined antiretroviral therapy era. AIDS 2010:24(10):1387-405
2. Barroso J, Voss JG. Fatigue in HIV and AIDS: an analysis of evidence. $J$ Assoc Nurses AIDS Care. 2013;24(1 Suppl):S5-14.

3. daCosta DiBonaventura M, Gupta S, Cho M, Mrus J. The association of HIV/AIDS treatment side effects with health status, work productivity, and resource use. AIDS Care. 2012:24(6):744-55.

4. Sowa NA, Bengtson A, Gaynes BN, Pence BW. Predictors of depression recovery in HIV-infected individuals managed through measurementbased care in infectious disease clinics. J Affect Disord. 2016;192:153-61.

5. O'Brien KK, Solomon P, Bergin C, et al. Reliability and validity of a new HIV-specific questionnaire with adults living with HIV in Canada and Ireland: the HIV disability questionnaire (HDQ). Health Qual Life Outcomes. 2015;13:124.

6. Simmonds MJ, Novy D, Sandoval R. The differential influence of pain and fatigue on physical performance and health status in ambulatory patients with human immunodeficiency virus. Clin J Pain. 2005;21(3):200-6.

7. Al-Dakkak I, Patel S, McCann E, Gadkari A, Prajapati G, Maiese EM. The impact of specific HIV treatment-related adverse events on adherence to antiretroviral therapy: a systematic review and meta-analysis. AIDS Care. 2013;25(4):400-14.

8. Marconi VC, Wu B, Hampton J, et al. Early warning indicators for first-line virologic failure independent of adherence measures in a South African urban clinic. AIDS Patient Care STDS. 2013;27(12):657-68.

9. Fredericksen RJ, Edwards TC, Merlin JS, et al. Patient and provider priorities for self-reported domains of HIV clinical care. AIDS Care. 2015;27(10):1255-64.

10. Barroso J, Leserman J, Harmon JL, Hammill B, Pence BW. Fatigue in HIV-infected people: a three-year observational study. J Pain Symptom Manag. 2015;50(1):69-79

11. Barroso J, Lynn MR. Psychometric properties of the HIV-related fatigue scale. J Assoc Nurses AIDS Care. 2002;13(1):66-75.

12. Pence BW, Barroso J, Leserman J, Harmon JL, Salahuddin N. Measuring fatigue in people living with HIV/AIDS: psychometric characteristics of the HIV-related fatigue scale. AIDS Care. 2008:20(7):829-37.

13. Justice AC, Holmes W, Gifford AL, et al. Development and validation of a self-completed HIV Symptom Index. J Clin Epidemiol. 2001;54(Suppl 1):S77-90.

14. Lai JS, Cella D, Choi S, et al. How item banks and their application can influence measurement practice in rehabilitation medicine: a PROMIS fatigue item bank example. Arch Phys Med Rehabil. 2011;92(10 Suppl):S20-7.

15. Cella D, Lai JS, Jensen SE, et al. Clinical validity of the PROMIS fatigue item bank across diverse clinical samples. J Clin Epidemiol. 2016;73:128-34.

16. Junghaenel DU, Christodoulou C, Lai JS, Stone AA. Demographic correlates of fatigue in the US general population: results from the patientreported outcomes measurement information system (PROMIS) initiative. J Psychosom Res. 2011;71(3):117-23.

17. Kitahata MM, Rodriguez B, Haubrich $R$, et al. Cohort profile: the centers for AIDS research network of integrated clinical systems. Int J Epidemiol. 2008;37(5):948-55.

18. Crane HM, Lober W, Webster E, et al. Routine collection of patientreported outcomes in an HIV clinic setting: the first 100 patients. Curr HIV Res. 2007;5(1):109-18.

19. Fredericksen RJ, Crane PK, Tufano J, et al. Integrating a web-based patient assessent into primary care for HIV-infected adults. J AIDS HIV Res. 2012:4(2):47-55.

20. Lawrence ST, Willig JH, Crane HM, et al. Routine, self-administered, touch-screen, computer-based suicidal ideation assessment linked to automated response team notification in an HIV primary care setting. Clin Infect Dis. 2010;50(8):1165-73.

21. Spitzer RL, Kroenke K, Williams JB. Validation and utility of a self-report version of PRIME-MD: the PHQ primary care study. Primary care evaluation of mental disorders. Patient health questionnaire. JAMA. 1999;282(18):1737-44.

22. Kroenke K, Spitzer RL, Williams JB. The PHQ-9: validity of a brief depression severity measure. J Gen Intern Med. 2001;16(9):606-13.

23. Newcombe DA, Humeniuk RE, Ali R. Validation of the World Health Organization alcohol, smoking and substance involvement screening test (ASSIST): report of results from the Australian site. Drug Alcohol Rev. 2005;24(3):217-26. 
24. WHO Assist Working Group. The alcohol, smoking and substance involvement screening test (ASSIST): development, reliability and feasibility. Addiction. 2002;97(9):1183-94.

25. Edwards TC, Fredericksen RJ, Crane HM, et al. Content validity of PatientReported Outcomes Measurement Information System (PROMIS) items in the context of HIV clinical care. Qual Life Res. 2016;25(2):293-302.

26. DeWalt DA, Rothrock N, Yount S, Stone AA. Evaluation of item candidates: the PROMIS qualitative item review. Med Care. 2007:45(5 Suppl 1):S12-21.

27. Garcia SF, Cella D, Clauser SB, et al. Standardizing patient-reported outcomes assessment in cancer clinical trials: a patient-reported outcomes measurement information system initiative. J Clin Oncol. 2007;25(32):5106-12.

28. Stata statistical software: release 14. [computer program]. College Station: StataCorp LP; 2015.

29. Mplus: statistical analysis with latent variables [computer program]. Version 7.11. Los Angeles: Muthén \& Muthén; 1998-2013.

30. Reeve BB, Hays RD, Bjorner JB, et al. Psychometric evaluation and calibration of health-related quality of life item banks: plans for the PatientReported Outcomes Measurement Information System (PROMIS). Med Care. 2007;45(5 Suppl 1):S22-31.

31. Choi SW. Firestar: computerized adaptive testing simulation program for polytomous item response theory models. Appl Psych Meas. 2009;33(8):644-5.

32. Crane PK, Gibbons LE, Jolley L, van Belle G. Differential item functioning analysis with ordinal logistic regression techniques: DIFdetect and difwithpar. Med Care. 2006;44(11 Suppl 3):S115-23.

33. Wu AW, Jacobson KL, Frick KD, et al. Validity and responsiveness of the euroqol as a measure of health- related quality of life in people enrolled in an AIDS clinical trial. Qual Life Res. 2002;11(3):273-82.

34. Johnson JA, Coons SJ. Comparison of the EQ-5D and SF-12 in an adult US sample. Qual Life Res. 1998;7(2):155-66.

35. Johnson JA, Coons SJ, Ergo A, Szava-Kovats G. Valuation of EuroQOL (EQ-5D) health states in an adult US sample. Pharmacoeconomics. 1998;13(4):421-33.

36. Harding R, Clucas C, Lampe FC, et al. What factors are associated with patient self-reported health status among HIV outpatients? A multi-centre UK study of biomedical and psychosocial factors. AIDS Care. 2012;24(8):963-71.

37. Chesney MA, Ickovics JR, Chambers DB, et al. Self-reported adherence to antiretroviral medications among participants in HIV clinical trials: the AACTG adherence instruments. Patient Care Committee \& Adherence Working Group of the Outcomes Committee of the Adult AIDS Clinical Trials Group (AACTG). AIDS Care. 2000;12(3):255-66.

38. Lu M, Safren SA, Skolnik PR, et al. Optimal recall period and response task for self-reported HIV medication adherence. AIDS Behav. 2008;12(1):86-94.

39. Walsh JC, Mandalia S, Gazzard BG. Responses to a 1 month self-report on adherence to antiretroviral therapy are consistent with electronic data and virological treatment outcome. AIDS. 2002;16(2):269-77.

40. Kalichman SC, Cain D, Fuhrel A, Eaton L, Di Fonzo K, Ertl T. Assessing medication adherence self-efficacy among low-literacy patients: development of a pictographic visual analogue scale. Health Educ Res. 2005;20(1):24-35.

41. Giordano TP, Guzman D, Clark R, Charlebois ED, Bangsberg DR. Measuring adherence to antiretroviral therapy in a diverse population using a visual analogue scale. HIV Clin Trials. 2004;5(2):74-9.

42. Feldman BJ, Fredericksen RJ, Crane PK, et al. Evaluation of the single-item self-rating adherence scale for use in routine clinical care of people living with HIV. AIDS Behav. 2012;17(1):307-18.

43. O'Brien KK, Davis AM, Strike C, Young NL, Bayoumi AM. Putting episodic disability into context: a qualitative study exploring factors that influence disability experienced by adults living with HIV/AIDS. J Int AIDS Soc. 2009;12:5.

44. McDonald RP. Test theory: a unified treatment. Mahwah: Lawrence Erlbaum; 1999.

45. Mungas D, Reed BR. Application of item response theory for development of a global functioning measure of dementia with linear measurement properties. Stat Med. 2000;19(11-12):1631-44.

46. Cook KF, Jensen SE, Schalet BD, et al. PROMIS measures of pain, fatigue, negative affect, physical function and social function demonstrate clinical validity across a range of chronic conditions. J Clin Epidemiol. 2016;73:89.

\section{Submit your next manuscript to BioMed Central and we will help you at every step:}

- We accept pre-submission inquiries

- Our selector tool helps you to find the most relevant journal

- We provide round the clock customer support

- Convenient online submission

- Thorough peer review

- Inclusion in PubMed and all major indexing services

- Maximum visibility for your research

Submit your manuscript at www.biomedcentral.com/submit
() Biomed Central 